\title{
Exercise Induced Atrioventricular Block in a Patient With Aortic Stenosis
}

\author{
Richard Good ${ }^{\mathrm{a}}$, Andrew R Chapman ${ }^{\mathrm{a}}$, Jamie AL Smith ${ }^{\mathrm{a}}$, Stephen J Leslie ${ }^{\mathrm{a}, \mathrm{b}, \mathrm{c}}$
}

\begin{abstract}
We present the case of a 62 year old gentleman with recurrent presyncopal episodes. During his first admission, an initial ECG revealed right bundle branch block and echocardiogram revealed moderate aortic stenosis, but no further precipitants were identified. A further episode prompted his readmission, and the patient requested to demonstrate symptoms whilst exercising. During exercise no symptoms were noted, but after a short period of rest, his symptoms appeared. ECG testing revealed second degree atrioventricular block (Mobitz type II), and a prolonged ventricular pause of almost six seconds. It is known that calcific aortic stenosis is associated with impaired atrioventricular node conduction, particularly when there is annular calcification. Whilst exercise testing is generally discouraged in symptomatic aortic stenosis, this case serves as a reminder that potentially treatable conduction disorders can be easily missed. Atrioventricular block should be considered when patients with aortic stenosis present with isolated presyncope. To our knowledge, this is the first case report to describe exercise induced transient AV node block in the presence of moderate to severe aortic stenosis.
\end{abstract}

Keywords: Aortic stenosis; Syncope; Atrioventricular block; Pacemaker; Exercise test

Manuscript accepted for publication December 7, 2011

${ }^{a}$ Cardiac Unit, Raigmore Hospital, Inverness IV2 3UJ, UK

${ }^{\mathrm{b}}$ University of Stirling, Highland Campus, Old Perth Road, Inverness, IV2 3JH, UK

${ }^{\mathrm{c}}$ Corresponding author: Stephen J Leslie, Consultant Cardiologist, Cardiac Unit, Raigmore Hospital, Inverness, IV2 3UJ, UK.

Email: stephen.leslie@nhs.net

doi:10.4021/jmc464w

\section{Introduction}

Aortic Stenosis is a condition characterised by abnormal narrowing of the aortic valve outlet. Whilst some patients may be asymptomatic, those with moderate to severe stenosis often present with exertional dyspnoea, chest pain, syncope and heart failure. Less commonly described complications of aortic stenosis include disorders of conduction. This case report describes a patient with previously undiagnosed aortic stenosis presenting with isolated presyncopal episodes.

\section{Case Report}

A 62 year old gentleman with no cardiac history was admitted with a 3 weeks history of recurrent presyncope. These episodes occurred on moderate exertion, lasted only a few seconds and resolved with rest. He denied symptoms of chest pain or breathlessness but did report an awareness of intermittent palpitations, which he described as a 'heavy thud in the chest,' mainly in bed at night.

Examination was generally unremarkable, with the exception of mild systolic hypertension $165 / 80 \mathrm{mmHg}$ and a soft, grade $3 / 6$, systolic murmur over the precordium radiating to the neck. Initial investigations including full blood count, electrolytes and thyroid function were normal. His resting ECG (Fig. 1) showed sinus rhythm with a normal axis. There was right bundle branch block morphology and the PR and QTc intervals were normal. The patient was admitted and monitored for 24 hours before being discharged with plans for outpatient echocardiography and Holter monitoring.

The patient was readmitted the following week with further presyncopal episodes on exertion. History and examination were unchanged. His baseline ECG was almost identical, except a prolonged PR interval of $210 \mathrm{~ms}$ was noted. Routine monitoring again revealed no abnormality. The patient suggested that he could try to replicate his symptoms through exercise. Under supervision, he walked briskly along the corridor without any symptoms. After two minutes rest, he had an episode of presyncope and an ECG confirmed 


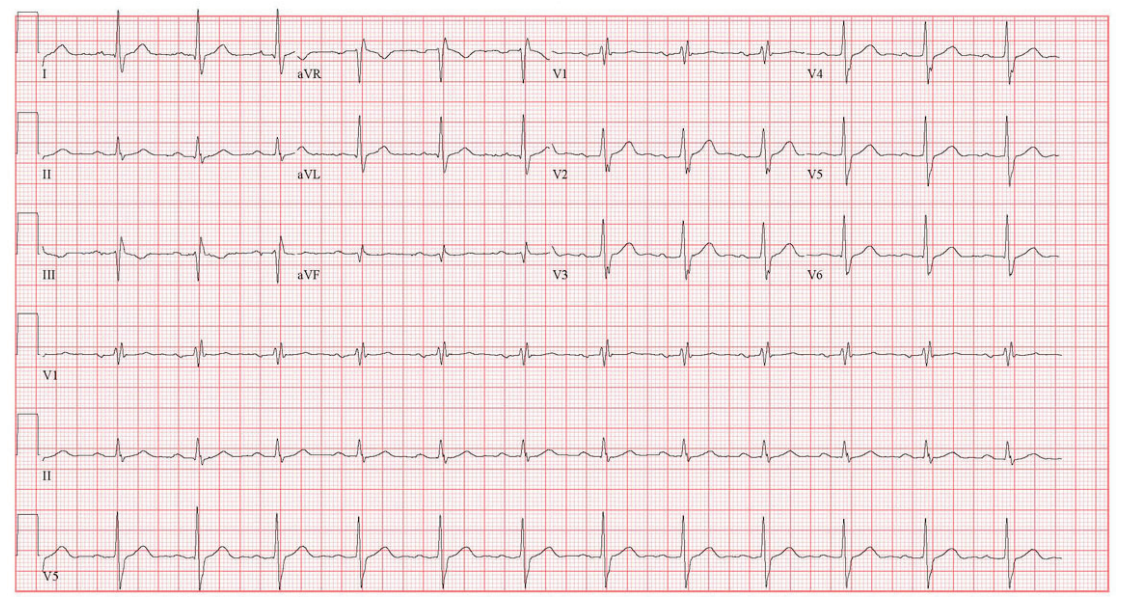

Figure 1. 12 lead ECG on initial presentation.

second degree atrioventricular block (Mobitz type II), and a prolonged ventricular pause of approximately 6 seconds (Fig. 2).

An urgent echocardiogram was arranged. The aortic valve was found to be moderately thickened and calcified with restricted opening. Continuous wave Doppler velocities suggested a peak gradient of $70 \mathrm{mmHg}$ in keeping with moderate to severe aortic stenosis. In addition, mild aortic regurgitation was noted. There was mild left ventricular hypertrophy but preserved systolic function. No other structural abnormalities were identified. Coronary angiography demonstrated only minor coronary artery disease.

A dual chamber pacemaker was implanted 2 days after admission. Atrial pacing at the time of pacemaker implan- tation confirmed 1:1 atrioventricular conduction up to 130 beats per minute. The patient was discharged the following day with plans for early review and reassessment of his aortic valve. He has had no further episodes of presyncope since discharge. His nocturnal palpitations have resolved and he can exercise freely without breathlessness or angina.

\section{Discussion}

Syncopal or presyncopal symptoms in patients with aortic stenosis are well recognised and thought to be caused by heightened left-ventricular baroreceptor activation. The development of these symptoms is an important marker of in-

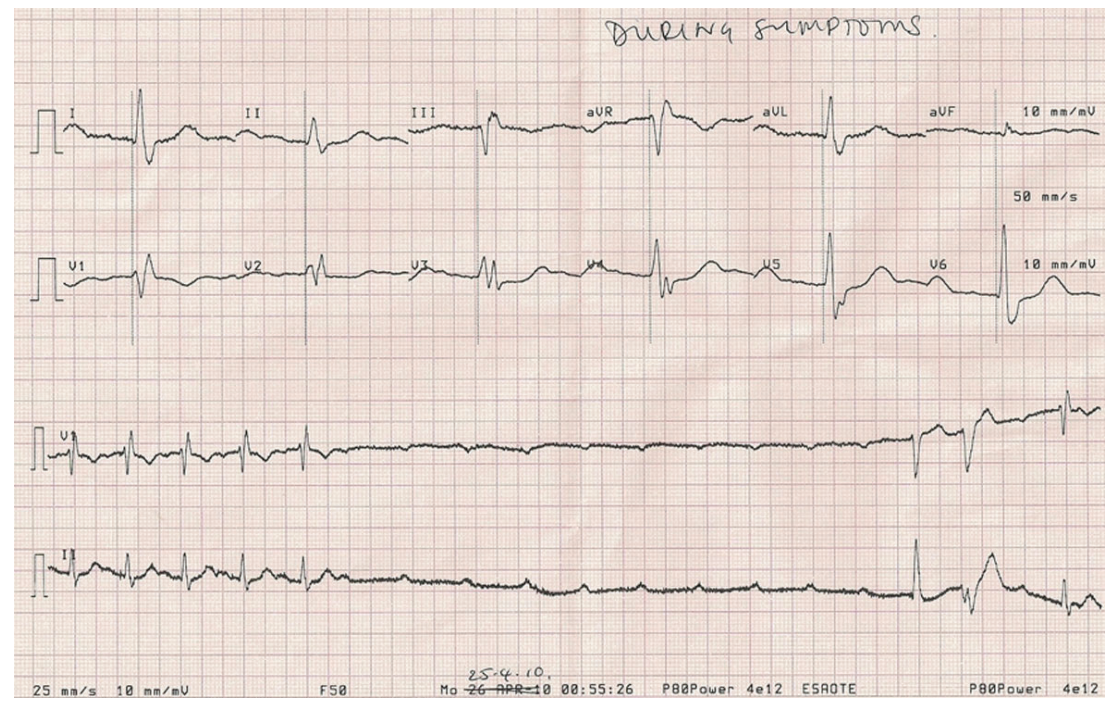

Figure 2. ECG recording 2 minutes after a brisk walk. 
creased mortality and often prompts referral for aortic valve replacement. However, calcific aortic valve disease is also associated with impaired atrioventricular node conduction, particularly in cases with marked annular calcification [1].

This case serves as a reminder to consider investigating patients with aortic valve stenosis for conduction disease if they present with isolated syncope or presyncope. This also raises the dilemma of when it is sensible to exercise patients with significant aortic stenosis. Generally, guidelines discourage formal exercise testing in symptomatic patients $[2$, 3], but, as we have demonstrated, this approach risks missing a significant and treatable alternative diagnosis.

\section{Conclusions}

To our knowledge, this is the first case in the literature to report presyncopal symptoms on exercise related to transient AV node block, in the presence of moderate to severe aortic stenosis.

\section{Conflict of Interest}

There are no conflicts of interest to be declared.

\section{References}

1. Friedman HS, Zaman Q, Haft JI, Melendez S. Assessment of atrioventricular conduction in aortic valve disease. Br Heart J. 1978;40(8):911-917.

2. Vahanian A, Baumgartner H, Bax J, Butchart E, Dion R, Filippatos G, Flachskampf F, et al. Guidelines on the management of valvular heart disease: The Task Force on the Management of Valvular Heart Disease of the European Society of Cardiology. Eur Heart J. 2007;28(2):230-268.

3. Das P, Rimington H, Chambers J. Exercise testing to stratify risk in aortic stenosis. Eur Heart J. 2005;26(13):1309-1313. 